\title{
ANÁlISE ESTATÍSTICA COMO SUPORTE AO PROCESSO DECISÓRIO SOBRE O FUNCIONAMENTO AOS SÁBADOS DE UMA BIBLIOTECA UNIVERSITÁRIA
}

\author{
STATISTICAL ANALYSIS SUPPORTING DECISION-MAKING ABOUT OPENING AN \\ UNIVERSITY LIBRARY ON SATURDAYS
}

ANÁLISIS ESTADÍSTICO COMO SOPORTE AL PROCESO DECISIVO SOBRE EL FUNCIONAMIENTO EN LOS SÁBADOS DE UNA BIBLIOTECA

${ }^{1}$ Lisandra Maria Kovaliczn Nadal, ${ }^{1}$ Claudia Tania Picinin, ${ }^{1}$ Maria Lúcia Cazarin Beserra Madruga

${ }^{1}$ Universidade Estadual de Ponta Grossa

\section{Correspondência}

${ }^{1}$ Lisandra Maria Kovaliczn Nadal

Universidade Estadual de Ponta Grossa

Ponta Grossa, PR

Email: lisandramkn@gmail.com

ORCID: http://orcid.org/0000-0003-1512-0745

Submetido em: 10-03-2017

Aceito em: 31-05-2017

Publicado: 07-06-2017

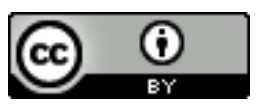

JITA: DD. Academic libraries 
RESUMO: A preocupação com o bem-estar dos funcionários e a redução significativa na demanda de empréstimo de livros aos sábados por alunos da pós-graduação levou à alteração dos dias de funcionamento das unidades de informação da Biblioteca Central Professor Faris Michaele (BICEN) da Universidade Estadual de Ponta Grossa (UEPG), no município de Ponta Grossa - PR. Logo, o estudo buscou suportar a decisão de fechamento da biblioteca universitária aos sábados em 2016. Verificou-se se há significância estatística na relação entre o tipo de usuário da biblioteca e a quantidade de exemplares emprestados aos sábados, e se o empréstimo de livros por alunos de pós-graduação era relevante em relação aos demais. Determinou-se, com base nos dados de empréstimo entre fevereiro de 2014 e dezembro de 2015, que há significância na relação entre o tipo de usuário da biblioteca e a quantidade de exemplares emprestados, e que o empréstimo de livros por alunos da graduação tem maior relevância em relação aos demais. Considerando ainda a economia de recursos como luz e horas extras e a manutenção do atendimento às normas do Ministério da Educação (MEC) para aprovação de cursos de graduação, o fechamento aos sábados durante o ano letivo de 2016 foi a decisão correta.

PALAVRAS-CHAVE: Biblioteca universitária. Estudo de usuário. Análise de dados.

ABSTRACT: The concern with the welfare of employees and the significant reduction in the demand of books loans by postgraduate students on Saturdays led to a change in operating days at the information units of Central Library Professor Faris Michaele (BICEN), in State University of Ponta Grossa (UEPG), in Ponta Grossa, PR. Therefore, the study intended to support the decision of closing the university library on Saturdays in 2016. It was verified whether there is statistical significance in the relationship between the type of library user and the number of books borrowed on Saturdays, and whether the loan of books by postgraduate students was relevant compared to others. Based on the loan data between February 2014 and December 2015, it was determined that there is a significant relationship between the type of library user and the number of borrowed books, and that the loan of books by undergraduate students is the most relevant. Also considering the saving of resources such as light and overtime and the maintenance of compliance with the norms of the Ministry of Education (MEC) for the approval of undergraduate courses, closing the units on Saturdays during the academic year of 2016 was the right decision.

KEYWORDS: University library. User study. Data analysis.

RESUMEN: La preocupación con el bienestar de los funcionarios y la reducción significativa de la demanda de préstamos de libros durante los sábados por alumnos del pos-grado llevó a la alteración de los días de funcionamiento de las unidades de información de la Biblioteca Central Profesor Faris Michaele (BICEN) de la Universidade Estadual de Ponta Grossa (UEPG), en el municipio de Ponta Grossa-PR. Luego, el estudio busca apoyar la decisión del cierre de la biblioteca universitaria durante los sábados en 2016. Fue verificado si hay una estadística relevante en la relación entre el tipo de usuario de la biblioteca y la cantidad de ejemplares prestados durante los sábados, y si el préstamo de libros por alumnos de pos-grado era relevante en relación a los demás. Se determinó con base en los datos de préstamo entre febrero de 2014 y diciembre de 2015, que hay importancia en la relación entre el tipo de usuario de la biblioteca y la cantidad de ejemplares prestados, y que el préstamo de libros por alumnos de grado tiene mayor importancia en relación a los demás. Considerando todavía la economía de recursos como luz y horas extras y el mantenimiento del tratamiento a las normas del Ministerio de Educación (MEC) para la aprobación de carreras de grado, el cierre durante los sábados del año lectivo 2016, fue una decisión correcta.

PALAVRaS ClaVE: Biblioteca universitaria. Estudio del usuario. Análisis de datos. 


\section{INTRODUÇÃ̃o}

A atualização e a ampliação das demandas informacionais nos últimos vinte anos induziram uma mudança de foco nas unidades de informação: da constituição e manutenção do acervo para a excelência no atendimento ao cliente. A biblioteca universitária precisa ser dinamizada para atrair toda a comunidade e garantir o atendimento às necessidades destes usuários plurais. Com esta finalidade, surgiram diversos estudos de usuário envolvendo questionários para averiguação dos serviços mais utilizados e da qualidade do atendimento ao público nas bibliotecas. A análise destes estudos permite o emprego de ferramentas de gestão e qualidade antes utilizadas apenas nos setores da indústria e do comércio, trazendo uma nova perspectiva às instituições públicas de ensino e exigindo uma maior capacitação dos funcionários. Um atendimento mais humano e especializado nas bibliotecas é um atrativo para gerações que têm acesso a muita informação porém sem muitas vezes o conhecimento necessário para empregá-la adequadamente.

A ideia de priorizar qualidade em relação a quantidade levou a uma reavaliação sobre o horário de funcionamento das unidades de informação da biblioteca universitária Biblioteca Central Professor Faris Michaele (BICEN) da Universidade Estadual de Ponta Grossa (UEPG), situada no município de Ponta Grossa - PR. Duas unidades de informação, Campus Central e Campus Uvaranas, abriam aos sábados no período da tarde durante quatro horas com o objetivo principal de atender aos discentes dos cursos de pós-graduação que assistiam aulas às sextas e sábados somente. Ao longo dos últimos dois anos observou-se, porém, uma diminuição significativa na demanda deste público, logo o presente estudo visa suportar a decisão de fechamento da biblioteca universitária aos sábados em 2016.

Determinou-se, com o auxílio de métodos estatísticos, se há diferença significante entre o tipo de usuário da biblioteca e a quantidade de exemplares emprestados aos sábados, e se o empréstimo de livros por alunos de pós-graduação é relevante em relação aos demais. Buscou-se assim apoio ao processo decisório da real necessidade de funcionamento aos sábados das unidades de informação da biblioteca universitária, levando em consideração o bem-estar dos funcionários, economia de recursos como luz e horas extras, e a manutenção do atendimento às normas do Ministério da Educação (MEC) para aprovação de cursos de graduação.

\section{BIBLIOTECA UNIVERSITÁRIA E O CONTEXTO ATUAL}

No século XXI, com a globalização e o fácil acesso a novas tecnologias, estamos a um toque de informações do mundo todo. No contexto das universidades, ocorre a dinamização dos estudos e parcerias, através de trabalhos, artigos científicos e livros publicados online, auxiliando os pesquisadores na construção do saber e minimizando a necessidade de arquivos físicos. Ainda assim, a biblioteca universitária é o principal recurso para facilitar o acesso à 
informação e deve ser co-participante nos projetos, atuando como agente mediador entre o conhecimento gerado e o usuário (SANTOS, 2012).

Para Girard e Girard (2013) e Mueller (1984), as bibliotecas universitárias vêm passando por uma transição de suma importância para a sociedade, apesar das funções básicas dos serviços continuarem sendo as mesmas. Como destaca Mueller (1984, p. 50):

As funções básicas de seus serviços têm permanecido e permanecerão as mesmas, isto é, a preservação, organização e difusão do conhecimento ou informação. O que tem mudado e continuará a mudar são as formas de desempenho, e o uso que é feito de suas atividades.

As mudanças são guiadas pelo maior acesso à informação, fruto dos meios digitais, que reforçam o papel das unidades de informação na intermediação do usuário com o conhecimento. $\mathrm{O}$ foco de suas atividades passa da manutenção de um acervo considerável para melhoria contínua no atendimento aos usuários. Mantém-se a importância do planejamento, aquisição, organização, controle e desenvolvimento de coleções, mas incorporam-se estudos de usuário para atuar eficazmente na qualidade dos serviços prestados (PALETTA et al., 2016).

Para um atendimento de qualidade, devemos levar em conta também o bem-estar dos funcionários que atuam junto aos usuários, respeitando valores, fornecendo treinamentos e carga horária adequada de trabalho. Meireles e Santos (2010) ressaltam que a automação dos sistemas fez com que a maior parte das tarefas diárias passassem a ser realizadas à frente do computador, somando-se a atividades relacionadas ao acervo, gerando maior necessidade de treinamentos e consequentemente aumentando a carga de trabalho. A qualidade de vida no trabalho pode ser definida pelo "equilíbrio entre satisfação do trabalhador, bem-estar, condições de trabalho para executar as tarefas, e a estabilidade financeira das organizações", de acordo com Oliveira e Mello (2016, p. 271). Este é um dos fatores que atrai pessoas de diversas faixas etárias para instituições públicas na atualidade.

Segundo Maculan et al. (2011, p. 4), "estabelecer uma cultura de bom atendimento demanda tempo e conhecimento das pessoas envolvidas nesse processo, envolve a afetividade delas e suas formas de relacionamento, e dependem dos gestores para se consolidar". A qualidade nos serviços, conforme tratam Silva e Rados (2002), pode ser percebida através de componentes tangíveis, como o nível de organização do ambiente, e intangíveis, como a cordialidade e a cooperação no atendimento.

Metodologias específicas devem embasar os estudos de usuário, para isso autores defendem o uso de métodos estatísticos, definindo variáveis quantitativas e qualitativas para tal. Estes estudos, quando criteriosos, trazem como resultado possibilidades de melhoria nos serviços prestados pela biblioteca e a satisfação do usuário final (METCHKO, 1981; LÜCK et al., 2000; PALETTA et al., 2016; MACULAN et al., 2011). 
Quaisquer implementos realizados devem, ainda, garantir o atendimento aos requisitos mínimos previstos pelo Ministério da Educação (MEC) para aprovação de cursos do ensino superior. Para a categoria de análise da biblioteca universitária, são levados em conta o espaço físico, o acervo, e os serviços oferecidos. No quesito horário de funcionamento da biblioteca universitária, o MEC exige que esta funcione em, pelo menos, dois turnos (incluído o do curso), sendo um deles noturno (BRASIL, 2002).

A combinação dos fatores deve garantir a satisfação de usuários e funcionários, transformando a relação entre a biblioteca universitária e a sociedade. Este é o principal objetivo das instituições de ensino.

\section{METODOLOGIA}

O processo decisório sobre o funcionamento do sistema de bibliotecas BICEN segue algumas premissas, ilustradas na Figura 1.

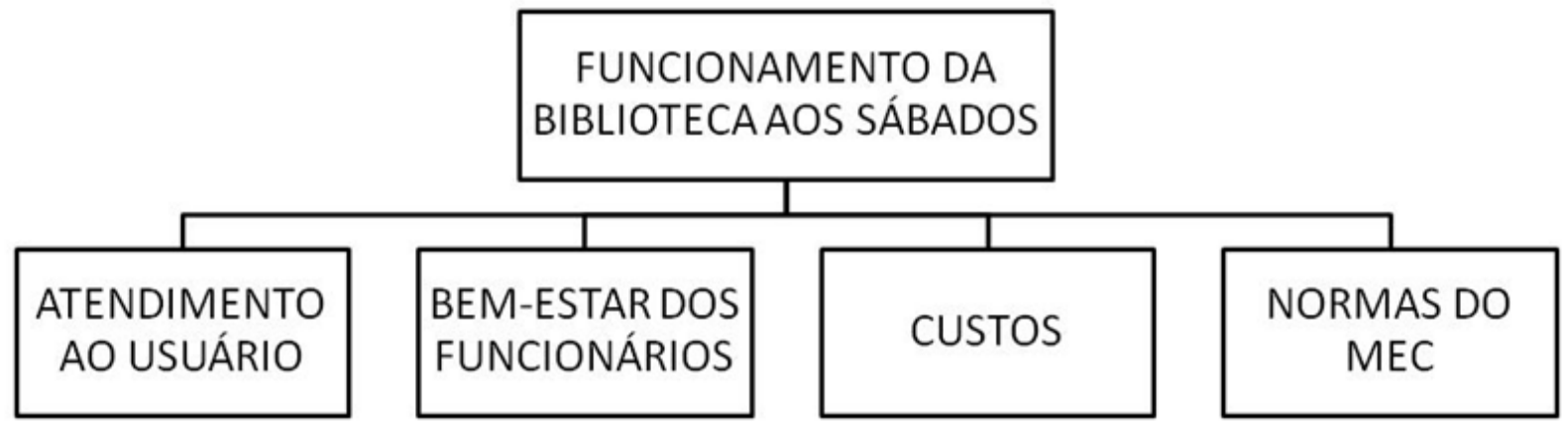

Figura 1. Ilustração do processo decisório sobre funcionamento da BICEN.

Fonte: Autoria própria (2016) com base no processo utilizado na BICEN.

O bem-estar dos funcionários foi avaliado apenas especulativamente, sem dados quantitativos ou qualitativos neste estudo. A garantia do atendimento às normas do MEC foi verificada considerando que as unidades continuem abertas de segunda a sexta das $8 \mathrm{~h} 00$ às $22 \mathrm{~h} 00$.

\subsection{Métodos estatísticos}

Como base de dados para análise estatística do atendimento ao usuário, foi utilizado um levantamento de número de exemplares - apenas livros - emprestados aos sábados entre fevereiro de 2014 e dezembro de 2015 nas bibliotecas Campus Central e Campus Uvaranas da UEPG, através de relatórios gerados no sistema Pergamum (PUCPR, 2016). 
A classificação para tipo de usuário empregada é apresentada na Tabela 1, sendo que o tipo 5 (Técnico Administrativo) engloba todos os funcionários da instituição e o tipo 6 (Outros usuários) toda a comunidade externa. Desta forma, podemos definir tipo de usuário como uma variável ordinal.

Tabela 1. Descrição dos tipos de usuário da biblioteca universitária da UEPG.

\begin{tabular}{cc}
\hline Tipo & Usuário \\
\hline 1 & Discente graduação \\
2 & Discente lato sensu \\
3 & Discente stricto sensu \\
4 & Docente \\
5 & Técnico Administrativo \\
6 & Outros usuários \\
\hline
\end{tabular}

Fonte: Autoria própria (2016) com base no sistema Pergamum (PUCPR, 2016).

O software IBM SPSS Statistics v.23 (Software Package Used for Statistical Analysis) recebeu os dados categorizados para a realização de análises estatísticas de (i) normalidade, (ii) correlação e (iii) variância. Primeiramente as variáveis foram categorizadas em qualitativas e quantitativas para seleção dos testes. Foi determinada a média de exemplares emprestados para cada tipo de usuário para definição dos que mais emprestaram no período em estudo.

\subsubsection{Testes de normalidade e correlação}

Para os testes de normalidade da variável quantitativa, verificou-se a presença de valores discrepantes, a assimetria e curtose, e a significância estatística pelo método de Shapiro-Wilk. Determinada a não-normalidade dos dados, na verificação da correlação entre o número de exemplares emprestados e o tipo de usuário foi empregado o teste nãoparamétrico do rô de Spearman, para determinar se há correlação, caso o nível de significância seja inferior a 95\%. Atestada a correlação, verificou-se o sinal (correlação positiva ou negativa) e o valor do rô para determinar se a relação é fraca (entre 0,0 e 0,4 ), moderada (entre 0,4 e 0,7 ) ou forte (entre 0,7 e 1,0 ).

\subsubsection{Testes de variância}

Para determinar se há diferença significativa entre os tipos de usuário, utilizou-se o teste de Kruskal-Wallis seguido de testes de Mann-Whitney par-a-par para determinar quais grupos de usuários apresentaram variância significativa de empréstimos entre si. O nível de significância adotado para os testes foi de $95 \%$.

Para o teste de Kruskal-Wallis, a hipótese nula (H0) considera que o tipo de usuário não afeta o número de exemplares emprestados aos sábados. Já a hipótese alternativa $(\mathrm{H} 1)$ considera que o tipo de usuário afeta o número de exemplares emprestados aos sábados.

\begin{tabular}{l|l|}
$\mathrm{v} .15$ & $\mathrm{n} .3$ \\
\hline
\end{tabular}

p.591-603
set./dez. 2017 


\subsection{Estimativa de custos}

$\mathrm{Na}$ avaliação dos custos considerou-se apenas os de maior relevância, que seriam:

a) energia elétrica: no cálculo estimado dos gastos com energia elétrica, os dados de quantidade de lâmpadas e computadores, bem como seu respectivo consumo, foram somados e multiplicados pelo custo por kWh pago pela instituição no mês de outubro de 2016, de acordo com a pró-reitoria responsável;

b) horas extras: para cálculo das horas extras, foi assumido um número de quatro técnicos em biblioteca para cada unidade de informação trabalhando aos sábados, com base no histórico, totalizando oito funcionários. O custo de cada hora extra foi determinado de acordo com a média entre os níveis 1 e 12 dos valores tabelados de salário-base 2016 para agente universitário nível médio classe 1 (funcionários com terceiro grau completo) na UEPG dividida por 180 horas e multiplicada por $150 \%$ (adicional de sábado de $50 \%$ ) (UNIVERSIDADE ESTADUAL DE PONTA GROSSA, 2016b).

A estimativa do custo total evitado no ano de 2016 pode ser obtida multiplicando os valores por hora de energia elétrica somada às horas extras pelas quatro horas aos sábados e pelos 34 sábados letivos de 2016, previstos no calendário universitário da UEPG (UNIVERSIDADE ESTADUAL DE PONTA GROSSA, 2016a).

\section{RESULTADOS E DISCUSSÕES}

Inicialmente, determinou-se que o número de exemplares emprestados aos sábados seria a variável quantitativa do estudo e o tipo de usuário (Tabela 1) a variável qualitativa ordinal. Após lançados os dados no software, foram obtidos os resultados para as análises que seguem.

\subsection{Análise das médias}

As médias do número de exemplares emprestados aos sábados em relação ao tipo de usuário são apresentadas na Tabela 2. 
Tabela 2. Média, número de ocorrências $(\mathrm{N})$ e desvio padrão (Desv. Pad.) de exemplares emprestados aos sábados de acordo com o tipo de usuário.

\begin{tabular}{|c|c|c|c|}
\hline Usuário & Média & $\mathbf{N}$ & Desv. Pad. \\
\hline Discente graduação & 13,45 & 102 & 7,971 \\
\hline Discente lato sensu & 3,86 & 7 & 1,574 \\
\hline Discente stricto sensu & 4,04 & 28 & 3,626 \\
\hline Docente & 2,91 & 22 & 2,348 \\
\hline Técnico Administrativo & 2,43 & 23 & 1,879 \\
\hline Outros usuários & 2,89 & 9 & 1,965 \\
\hline Total & 8,68 & 191 & 7,958 \\
\hline
\end{tabular}

Fonte: Autoria própria (2016).

Considerando-se apenas as médias de exemplares emprestados, os discentes da graduação apresentaram uma quantidade aproximadamente duas vezes maior do que os alunos da pós-graduação stricto e lato sensu juntos. Além disso, o número de ocorrências de empréstimo dos discentes da graduação (102 ocorrências) representou mais da metade do total nos dois anos (191 ocorrências), indicando que, além de emprestarem mais exemplares, também foram mais vezes até a biblioteca.

\subsection{Testes de normalidade e correlação}

Os resultados dos testes de normalidade (Tabela 3) apontam que o número de exemplares emprestados aos sábados no período de fevereiro de 2014 a dezembro de 2015, correspondem a dados não-normais, portanto justifica-se o uso de testes não-paramétricos nas análises.

Tabela 3. Resultados dos testes de normalidade para número de exemplares.

\begin{tabular}{lccc}
\hline & Real & $\begin{array}{c}\text { Padrão } \\
\text { normalidade }\end{array}$ & Conclusão \\
\hline Número de casos (N) & 191 & $>30$ & Normal \\
Valores discrepantes & 5 & 0 & Não-normal \\
Assimetria & 1,501 & $<|1,5|$ & Não-normal \\
Curtose & 2,653 & $<|1,5|$ & Não-normal \\
Teste Shapiro-Wilk (Sig.) & $<0,001$ & $>0,05$ & Não-normal \\
\hline
\end{tabular}

Fonte: Autoria própria (2016).

O teste de correlação de Spearman (Tabela 4) apresentou $\mathrm{p}<0,001$, o que indica que há correlação entre as variáveis quantitativa e qualitativa.

Tabela 4. Dados do teste não-paramétrico de correlação.

\begin{tabular}{lccc}
\hline & rô de Spearman & Sig. (bilateral) & N \\
$\mathrm{N}^{\mathrm{o}}$ de exemplares x tipo de usuário & $-0,737$ & $<0,001$ & 191 \\
\hline
\end{tabular}

Fonte: Autoria própria (2016).

Esta correlação é considerada forte (rô de Spearman superior a 0,7), indicando que o número de exemplares emprestados está relacionado ao tipo de usuário que realizou o

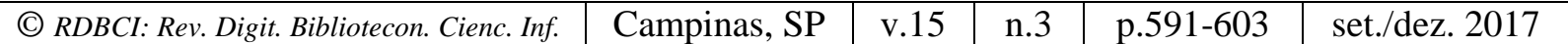


empréstimo. Isto demonstra que é válido considerar estas variáveis na tomada de decisão sobre o funcionamento da biblioteca universitária aos sábados.

Observa-se ainda que a correlação tem valor negativo, logo altos valores de número de exemplares emprestados tendem a se relacionar a baixos valores de tipo de usuário. Este fato corrobora com a análise das médias que indicou que usuários tipo 1 - discentes graduação emprestaram maior número de exemplares.

\subsection{Testes de variância}

Após verificada a forte correlação entre as variáveis, empregou-se o teste de KruskalWallis para determinar se há diferença significativa entre as médias, ou seja, se deve-se aceitar ou rejeitar a hipótese nula (H0) de que o tipo de usuário não afeta o número de exemplares emprestados aos sábados. Conforme resultados apresentados na Tabela 5, considerando $\mathrm{p}$ valor de 0,05 pode-se rejeitar $\mathrm{H} 0$.

Tabela 5. Resultado do teste não-paramétrico de variância.

\begin{tabular}{lccc}
\hline & Qui-quadrado & Sig. (bilateral) & gl \\
$\mathrm{N}^{\mathrm{o}}$ de exemplares x tipo de usuário & 108,173 & $<0,001$ & 5 \\
\hline
\end{tabular}
Fonte: Autoria própria (2016).

Para determinar entre quais tipos de usuário essa diferença de médias é significativa, aplicou-se o teste de Mann-Whitney com resultados de nível de significância indicados na Tabela 6.

Tabela 6. Níveis de significância obtidos a partir do teste de Mann-Whitney conforme os tipos de usuário comparados.

\begin{tabular}{|c|c|c|c|c|c|c|}
\hline Usuário & $\begin{array}{c}\text { Discente } \\
\text { graduação }\end{array}$ & $\begin{array}{l}\text { Discente } \\
\text { lato sensu }\end{array}$ & $\begin{array}{c}\text { Discente } \\
\text { stricto sensu }\end{array}$ & Docente & $\begin{array}{l}\text { Técnico } \\
\text { Admin. }\end{array}$ & $\begin{array}{l}\text { Outros } \\
\text { usuários }\end{array}$ \\
\hline $\begin{array}{c}\text { Discente } \\
\text { graduação }\end{array}$ & & $<0,001$ & $<0,001$ & $<0,001$ & $<0,001$ & $<0,001$ \\
\hline $\begin{array}{l}\text { Discente lato } \\
\quad \text { sensu }\end{array}$ & & & 0,476 & 0,094 & 0,025 & 0,206 \\
\hline $\begin{array}{l}\text { Discente stricto } \\
\text { sensu }\end{array}$ & & & & 0,195 & 0,051 & 0,460 \\
\hline Docente & & & & & 0,579 & 0,839 \\
\hline Técnico Admin. & & & & & & 0,514 \\
\hline Outros usuários & & & & & & \\
\hline
\end{tabular}

Fonte: Autoria própria (2016). 
Verifica-se que os discentes da graduação têm uma diferença significativa de médias quando comparados com todas as demais categorias, enquanto alunos de pós-graduação lato sensu só a possuem quando comparados aos funcionários da instituição. Esta inferência corrobora com os valores médios de exemplares emprestados por tipo de usuário, concluindose que os discentes da graduação têm uma expressiva representatividade quando comparados às demais categorias de usuários.

A partir dos resultados das análises estatísticas das médias, da correlação e da variância verifica-se que a categoria de usuário que mais utilizou as duas unidades de informação para empréstimo de livros foi a dos alunos de graduação da UEPG, quebrando-se assim o paradigma de que a biblioteca universitária deveria abrir aos sábados para atender a demanda dos alunos de pós-graduação. Um dos fatores que mais contribui para um menor número de empréstimos é o período de permanência com o livro, que é de uma semana para alunos da graduação, podendo estender-se por até seis semanas, e de um mês para alunos da pósgraduação, podendo estender-se por até seis meses. Além disso os alunos de pós-graduação têm direito a empréstimo de até oito exemplares por vez, enquanto os acadêmicos da graduação podem emprestar apenas quatro. Loynes e Proctor (2000), em seu estudo do impacto da redução do horário de funcionamento em bibliotecas públicas, destacam que normalmente esta redução é muito pequena para isolar das diversas variáveis que levam os alunos a frequentarem menos as bibliotecas.

Considerando que poucos alunos da pós-graduação fazem uso da biblioteca aos sábados, e que estes cursos ocorrem também às sextas-feiras, os discentes têm possibilidade de utilizar os serviços durante a semana, no contraturno dos seus cursos, visto que ambas as unidades ficam abertas nos períodos da manhã, da tarde e da noite. Kapoor (2010) observa que com a abertura das bibliotecas após as aulas e durante o horário de almoço os alunos tendem a utilizar mais esses períodos para consultar o acervo e emprestar livros, aumentando a frequência de usuários. Assim o quesito serviços da biblioteca universitária das normas do MEC para aprovação dos cursos continua sendo atendida, uma vez que se dá a oportunidade ao aluno de estudar no turno de funcionamento do seu curso e em outros horários, inclusive à noite (BRASIL, 2002).

O fechamento das bibliotecas Campus Central e Uvaranas aos sábados durante o ano letivo de 2016 evitou um custo estimado de $\mathrm{R} \$ 38.637,95$, considerando 34 sábados letivos com oito técnicos em biblioteca trabalhando a $\mathrm{R} \$ 33,96 / \mathrm{h}$ e um consumo de energia elétrica de $19,44 \mathrm{~kW} / \mathrm{h}$. Este não é um valor exato pois os salários variam de acordo com a formação e o tempo de casa dos funcionários, assim como o custo e consumo da energia variam ao longo do ano. O valor, porém, é representativo e constitui um fator que reforça a análise estatística, apontando a desvantagem econômica gerada caso a biblioteca abrisse aos sábados. 


\section{CONCLUSÕES}

Uma série de fatores contribui para um atendimento de qualidade e o bem-estar dos funcionários é um destes. Alinhando a redução na demanda dos usuários e a manutenção do descanso dos funcionários, o sistema de bibliotecas universitárias da UEPG optou por não funcionar mais aos sábados baseando sua decisão nos fatores de atendimento ao usuário, redução de custos e cumprimento das normas do MEC. A análise estatística dos dados de empréstimo de livros aos sábados entre fevereiro de 2014 e dezembro de 2015 demonstrou que existe relação entre o tipo de usuário e o número de exemplares emprestados. Mais da metade dos empréstimos no período foi realizado por alunos da graduação, contrariando a ideia de que os discentes stricto e lato sensu necessitam mais da biblioteca aos finais de semana. Visto que discentes da graduação podem utilizar os serviços durante a semana, em três turnos, as normas do MEC para aprovação de cursos segue atendida. Sendo assim o fechamento aos sábados durante o ano letivo de 2016 foi a decisão correta, que gerou ainda um custo evitado de cerca de $\mathrm{R} \$ 38.637,95$. Neste estudo foram utilizados apenas dados de empréstimo de livros aos sábados, desconsiderando usuários que utilizam o ambiente da biblioteca para fins de estudo e consulta local. Estudos de opinião dos usuários e dos funcionários da instituição sobre a decisão tomada e o impacto na qualidade do atendimento, através de questionários por exemplo, poderiam contribuir para uma análise mais aprofundada.

\section{REFERÊNCIAS}

BRASIL. Ministério da Educação. Secretaria de Educação Superior. Manual de verificação in loco das condições institucionais: credenciamento de instituições não universitárias; autorização de cursos superiores (ensino presencial e a distância). Brasília: MEC; SESu, 2002. 85 p. Disponível em: 〈http://portal.mec.gov.br/sesu/arquivos/pdf/Manual1.pdf>. Acesso em: 09 nov. 2016.

GIRARD, Carla Daniella Teixeira; GIRARD, Cristiane Marina Teixeira. A importância da biblioteca universitária como mediadora do processo de ensino-aprendizagem na educação superior: um estudo de caso da Biblioteca Paulo Freire da UEPA. Múltiplos Olhares em Ciência da Informação, Belo Horizonte, v. 3, n. 2, 2013. Disponível em: <http://portaldeperiodicos.eci.ufmg.br/index.php/moci/article/viewFile/1996/1420>. Acesso em: 09 nov. 2016.

IBM Corp. IBM SPSS Statistics for Windows, v. 23.0. Armonk: IBM Corp, [S.I.]. Software para análise estatística.

KAPOOR, Kanta. Print and electronic resources: usage statistics at Guru Gobind Singh Indraprastha University Library, Program, [S.1.], v. 44, n. 1, p.59-68, fev. 2010. Disponível em: 〈http://dx.doi.org/10.1108/00330331011019690〉. Acesso em: 10 nov. 2016.

LOYNES, Robert; PROCTOR, Richard. The effect of reductions in public library opening hours on book issues: a statistical analysis. Journal of Documentation, [S.1.], v. 56, n. 6 ,

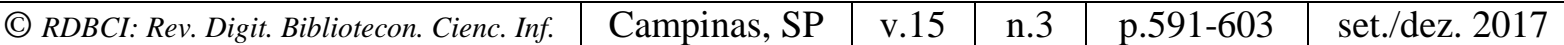


p.605-623, dez. 2000. Disponível em: 〈http://dx.doi.org/10.1108/eum0000000007129>. Acesso em: 10 nov. 2016.

LÜCK, Esther Hermes et al. A Biblioteca Universitária e as diretrizes curriculares do ensino de graduação. In: SEMINÁRIO NACIONAL DE BIBLIOTECAS UNIVERSITÁRIAS, 11., Florianópolis, 2000. Anais... Disponível em:

<http://snbu.bvs.br/snbu2000/docs/pt/doc/t024.doc>. Acesso em: 01 nov. 2016.

MACULAN, Benildes Coura Moreira dos Santos et al. Levantamento de usos e usuários: biblioteca do curso de biblioteconomia da Universidade Federal de Minas Gerais. Múltiplos Olhares em Ciência da Informação, Belo Horizonte, v. 1, n. 1, mar. 2011. Disponível em: <http://portaldeperiodicos.eci.ufmg.br/index.php/moci/article/viewFile/1223/823 >. Acesso em: 09 Nov. 2016.

MEIRELES, Maria Lucia Araujo; SANTOS, Jussara Pereira. Tecnologias da informação e a saúde dos funcionários das bibliotecas do campus da saúde da UFRGS: avaliação da necessidade de implantação de um programa de ginástica laboral. In: Seminário Nacional de Bibliotecas Universitárias, 16., 2010, Rio de Janeiro. Anais eletrônicos... Rio de Janeiro: UFRJ, 2010. Disponível em: 〈http://www.sibi.ufrj.br/snbu2010/pdfs/posters//final 111.pdf>. Acesso em: 09 nov. 2016.

METCHKO, Dulce Maria Bastos. Demandas de usuários de bibliotecas: revisão de literatura. Educ. rev., Curitiba, n.1, p.75-107, dez. 1981. Disponível em:

<http://www.scielo.br/scielo.php?script=sci arttext\&pid=S0104-40601981000100006>. Acesso em: 09 nov. 2016.

MUELLER, Suzana Pinheiro Machado. Bibliotecas e sociedade: evolução da interpretação de função e papéis da biblioteca. R. Esc. Bibliotecon. UFMG, Belo Horizonte, v. 13, n. 1, p. 7-54, mar. 1984. Disponível em: 〈http://basessibi.c3sl.ufpr.br/brapci/v/a/2698>. Acesso em: 09 Nov. 2016.

OLIVEIRA, Eunice Câmara de; MELLO, Josiane. Qualidade de vida no trabalho: um estudo realizado numa Biblioteca Universitária no Rio Grande do Norte. Revista ACB, [S.1.], v. 21, n. 2, p. 270-293, set. 2016. Disponível em:

<https://revista.acbsc.org.br/racb/article/view/1147>. Acesso em: 09 nov. 2016.

PALETTA, Francisco Carlos et al. Estudos de usuário e o planejamento dos serviços de informação em biblioteca. Revista ACB, [S.1.], v. 21, n. 1, p. 145-155, mar. 2016. Disponível em: 〈https://revista.acbsc.org.br/racb/article/view/1043>. Acesso em: 09 nov. 2016.

PUCPR. Pergamum v. 9 s. 0. Curitiba: Associação Paranaense de Cultura da PUCPR, 2016. Sistema informatizado de gerenciamento de dados.

SANTOS, Marivaldina Bulcão dos. Biblioteca universitária: acesso à informação e conhecimento. In: SEMINÁRIO NACIONAL DE BIBLIOTECAS UNIVERSITÁRIAS, 17., 2012, Gramado. Anais eletrônicos... Gramado: [S.I.], 2012. Disponível em:

<http://www.snbu2012.com.br/anais/pdf/4QHV.pdf>. Acesso em: 09 nov. 2016. 
SILVA, Chirley Cristiane Mineiro da; RADOS, Gregório Jean Varvakis. Gestão de serviços em bibliotecas: melhoria com foco no cliente. Revista ACB, [S.1.], v. 7, n. 2, p. 198-218, 2002. Disponível em: 〈https://revista.acbsc.org.br/racb/article/view/370>. Acesso em: 09 nov. 2016.

UNIVERSIDADE ESTADUAL DE PONTA GROSSA. Calendário da Universidade Estadual de Ponta Grossa para o ano de 2016. Disponível em:

<http://sites.uepg.br/prograd/wp-content/uploads/2013/09/Resolu\%C3\%A7\%C3\%A3041.pdf>. Acesso em: 21 nov. 2016a.

UNIVERSIDADE ESTADUAL DE PONTA GROSSA. Tabela de vencimento básico e remuneração carreira técnica universitária - IEES. Disponível em:

$<$ https://sistemas.uepg.br/producao/proreitorias/prorh/sistemasproreitoria/PortalServidor/paginas/MenuEsquerdo/CarreirasSalarios/c orpo_tec administrativo/tabelasalarial2.php>. Acesso em: 09 nov. $2016 \mathrm{~b}$.
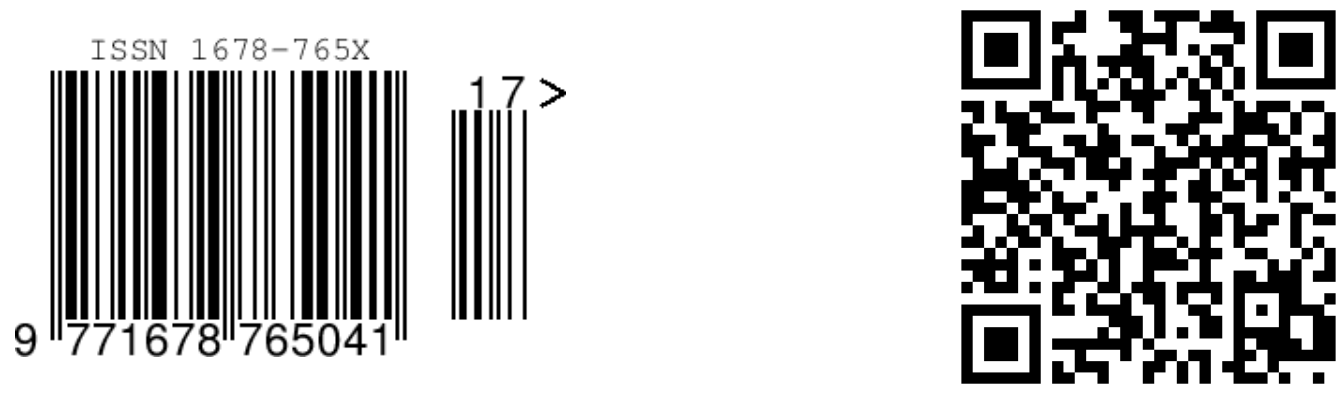